\title{
Problemas de la evaluación del impacto ambiental en relación al planteamiento territorial y urbanístico ${ }^{1}$
}

\author{
angel Paniagua Mazorra y Olga P. Tarancón Rico ${ }^{2}$
}

\section{INTRODUCCIÓN}

La tradicional tendencia a la concentración de la población en las ciudades, como consecuencia de los cambios tecnológicos en el medio rural y la expulsión que ello conlleva, así como las ventajas comparativas que ofrecen las aglomeraciones urbanas en materia de empleo, ha provocado una pérdida de la calidad medio ambiental y de las condiciones mínimas de vida para un porcentaje importante de la población mundial (ENTORNO 1991).

Por otro lado, los sistemas urbanos son "devoradores" de suelo y paisaje, por la enorme cantidad de desechos que deben ser metabolizados por el entorno, el cual ya sufre el impacto de la demanda energética y de los materiales. Los sistemas urbanos se convierten en sistemas altamente impactantes y sus zonas de afluencia se extienden desde su entorno inmediato hasta puntos lejanos (COMISIÓN DE LAS COMUNIDADES Europeas 1990, AleXANDre, A. 1992).

Al deteriorarse el medio natural inevitablemente se destruye el entorno humano puesto que son dos ámbitos estrechamente vinculados y en difícil equilibrio, dada la fragilidad de los ecosistemas frente a las agresiones de los sistemas industriales y urbanos (YOLDI, L. 1990).

Así, ante el fracaso de las teorías urbanísticas de tipo estático y de zonificación son necesarios los estudios que analizan el ecosistema ur-

1 Este artículo procede del estudio titulado «Evaluación de Impacto Ambiental y su aplicación en la planificación territorial» financiado por el instituto para el Territorio y el Urbanismo (MOPT).

2 Geógrafos. Universidad de León y Universidad Autónoma de Madrid, respectivamente. 
bano de manera dinámica, con enfoques conceptuales y metodológicos que reflejen la significación de las ciudades en relación al impacto que ejercen sobre el ambiente natural, que tomen en consideración la relación entre el desarrollo social y el ambiente, estilos de vida y la salud y el bienestar de la población humana (tanto urbana como rural), en definitiva, estudios de las interrelaciones y flujos entre el sistema urbano y su entorno, teniendo en cuenta que el ecosistema urbano es un sistema abierto y que por consiguiente la relación entre la ciudad y su entorno próximo y distante, en particular, el medio rural, debe constituir una preocupación esencial a la planificación y a la gestión de la tierra y de los recursos, como propone el Programa MAB de la UNESCO (MAB 1988).

En la misma línea por la preocupación medio ambiental y la calidad de vida se encuentran los trabajos realizados desde el Consejo de Europa, la O.C.D.E. y otras instancias administrativas; todas ellas coinciden en que una de las mayores dificultades a la hora de sopesar las preocupaciones por el medio ambiente y por el desarrollo económico está relacionada la escala temporal. Con demasiada frecuencia la gestión del medio ambiente responde a crisis a corto plazo y no emprende una planificación a largo plazo, que sería más económica.

Por ello, la Carta Europea de la Ordenación del Territorio define:

"La ordenación del territorio es la expresión espacial de las políticas económica, social, cultural y ecológica de cualquier sociedad.

Es a la vez una disciplina científica, una técnica administrativa y una política concebida como una actuación interdisciplinaria y global cuyo objetivo es un desarrollo equilibrado de las regiones y la organización física del espacio de acuerdo con unas directrices".

La Administración es la encargada de gestionar la planificación racional del medio ambiente, para ello se debe servir de la ordenación del territorio, del urbanismo o de la planificación rural; pero, frente a una planificación sectorial debe optar por la planificación integrada como una estrategia de conjunto, tal y como disponen las recomendaciones del Programa MAB, de la O.C.D.E. y de la Comunidad Económica Europea, utilizando para ello la técnica de la Evaluación de Impacto Ambiental (EIA).

En el presente trabajo se investiga, analiza y reflexiona los puntos mencionados anteriormente y la ayuda que puede aportar la incorporación de la técnica de evaluación de impacto ambiental a la ordenación del territorio, el urbanismo y la planificación rural. 


\section{PRINCIPIOS COMUNITARIOS EN MATERIA DE MEDIO AMBIENTE}

\subsection{Programas de Acción}

En los Tratados fundacionales de la CEE no se hacía mención específica a los problemas del medio ambiente. Pero, ante la demanda de una mejor calidad de vida, el análisis de los efectos de la industrialización y de la urbanización en 1970 se redacta el primer documento de la Comunidad que constituye la primera comunicación sobre la política de la Comunidad en materia de medio ambiente. Este documento, tras la cumbre de París de octubre de 1972, dio lugar al I Programa de Acción de las Comunidades Europeas sobre medio ambiente (1973-1976).

En el Primer Programa de 1973 se definieron los principios fundamentales de la política comunitaria en materia del medio ambiente, constituyéndose como constantes para los siguientes programas, incluso para el Acta Única Europea.

Son trece los principios en los que se inspira, de los que aquellos más relevantes son: 1 . La mejor política de medioambiente consiste más en evitar, el origen de la contaminación y los daños, antes que la posterior acción para combatir sus efectos. 2. La política del medio ambiente puede y debe ir sincronizada con el desarrollo económico y social. 3. Conviene tener en cuenta la incidencia de todos los procesos técnicos de planificación y de decisión sobre el medio ambiente. 4. Los aspectos más importantes de la política de medio ambiente nunca deben programarse ni realizarse de forma aislada por los diversos países. 5. La política comunitaria del medio ambiente debe tener por objeto, en tanto en cuanto sea posible, progresar de forma coordinada y armonizada con las políticas sectoriales.

Todos estos principios han sido y son los elementos rectores de la política ambiental comunitaria, aunque en el IV Programa de Acción han sido redefinidos.

De acuerdo con tales principios, las medidas de actuación que se proponen en el primer programa además de la reducción y prevención de la contaminación, se dirigen a: salvaguardar el medio rural, sobre todo desarrollando prácticas agrarias conformes con el cuidado del modelado y conservación del paisaje; evitar el agotamiento progresivo de determinados recursos naturales; en urbanismo y ordenación del territorio se plantean los desequilibrios regionales que están teniendo lugar, debido a la polarización entre rápida urbanización de zonas prósperas y abandono de zonas marginales; mejora del medio ambiente laboral; creación de una 
fundación europea para la mejora de las condiciones del trabajo y de vida; sensibilización ante la problemática ambiental.

En este primer plan se introduce una visión cualitativa del medio ambiente en relación a la calidad de vida, rompiendo los esquemas cuantitativos.

El Segundo Programa de Acción (1977-1981) fue aprobado en mayo de $1977^{3}$ en el mismo se pretende dotar de continuidad a la política iniciada en el Primer Programa, reafirmando sus principios y objetivos; a la vez que se concede una importancia particular a las medidas que creen mecanismos de acción preventiva, especialmente frente a la contaminación y la ordenación del territorio y el tratamiento de residuos; $y$, por último, es objeto de especial atención la protección y la gestión racional del espacio y de los recursos naturales.

En este sentido, se potencian las evaluaciones de impacto ambiental para conocer a priori las repercusiones de los procesos técnicos de planificación y de decisión sobre el medio ambiente.

Dentro de las actuaciones de este II Programa de Acción destaca la reducción de contaminación y daños, y, sobre todo la protección y gestión racional del espacio, del medio y de los recursos naturales, que abarca tres grandes secciones: establecimiento de un método de cartografía ecológica; acciones relativas al espacio rural y la silvicultura, así como problemas específicos de espacios urbanos y rurales, zonas de montaña y zonas costeras.

En conjunto, en este segundo programa no aparecen los grandes principios del primero, siendo más pragmático y concreto aunque no presenta ningún plazo concreto para conseguir objetivos.

El Tercer Programa de Acción (1983-1986) ${ }^{4}$ se caracteriza por tener una línea de continuidad con los dos primeros pero, sobre todo, por insistir en la necesidad de prevención en la política de medio ambiente. En este sentido, es fundamental el Reglamento CEE n. ${ }^{\circ} 1872 / 84$ del Consejo de 28 de junio de 1984 relativo a acciones comunitarias de medio ambiente que dota de apoyo financiero a los proyectos dirigidos al desarrollo de nuevas tecnologías limpias, de medición y calidad del medio ambiental natural y los tendentes a contribuir al mantenimiento o restablecimiento de biotipos amenazados.

3 DOCE, n. ${ }^{\circ} 139 / 46$ del 13 de junio de 1977.

4 Su aprobación se recoge en el Diario Oficial de las Comunidades Europeas, $n .{ }^{\circ} 46 / 1$ de 17 de febrero de 1983 
Ambos cristalizan en dos acciones básicas de este Programa: necesidad de integrar una dimensión medioambiental en las demás políticas. Incorpora, en este sentido, la importancia de los aspectos económicos y sociales en la política ambiental, resaltando su posible contribución a la resolución del desempleo. A la vez se destaca el papel de la política ambiental como una verdadera política estructural de continuidad a largo plazo, independientemente del momento coyuntural. Avanza en la necesidad de una integración del medio ambiente en el resto de políticas comunitarias; en segundo lugar se fomenta la utilización de la técnica o procedimiento de evaluación de repercusiones en el medio ambiente. Ello tuvo su plasmación en la Directiva 85/337/CEE del 25 de junio de 1985 sobre "Evaluación de los impactos sobre el medio ambiente de ciertas obras públicas y privadas".

En suma, el medio ambiente toma en este III Programa de Acción nuevas dimensiones:

Ya que se deberá tomar en consideración en el ámbito de los sectores económicos: agricultura, industria, transportes, turismo, energía,..., y, como afirma, LóPEZ A. (1990) dentro de esa estrategia global tendrán que realizarse las matizaciones regionales dadas las diferencias existentes entre las distintas regiones de la Comunidad Europea. Se reconoce la dificultad de realizar una política ambiental comunitaria dado que el principal instrumento de la política de medio ambiente ha sido la utilización de directivas. Su aplicación necesita de adaptación de la legislación nacional. En este proceso se había puesto de manifiesto el riesgo de una aplicación indebida.

En este estado de cosas, la mayor parte de los Estados miembros buscan inevitablemente minimizar los cambios a introducir en sus propios procedimientos para hacerlos compatibles con las directivas. De esta manera, cada directiva es interpretada desde una perspectiva particular. En ciertos casos, el resultado presenta una divergencia notoria (ETAT 1987).

Por otro lado, se remarca la necesidad de una protección y gestión racional del espacio, del medio y de los recursos naturales.

Por último, se refuerza la necesidad de información y concienciación, tanto al público en general como a los agentes socioeconómicos que actúan en el espacio, privados o públicos.

La preocupación por la protección del medio ambiente es esencial en la Comunidad Económica Europea desde la entrada en vigor del Acta Única Europea, a partir del 1 de julio de 1987; que ha supuesto la institucionalización que la C.E.E. seguía desde 1972. 
Sus amplios objetivos se encuentran recogidos en la Política del Primer Programa de Acción Comunitario, adoptado el 22 de noviembre de 1973, permaneciendo hasta el vigente (Cuarto Programa de Acción, adoptado el 19 de octubre de 1987).

Las disposiciones del Acta Única harán de la protección del medio ambiente un componente de las demás políticas de la Comunidad; considerando que la calidad del medio ambiente representa un beneficio, tanto en términos económicos como de bienestar. Por ello, la integración de las consideraciones ambientales en todas las decisiones económicosociales, es un principio recogido en el Tercer Programa de Acción; esta integración permitirá la compatibilidad entre el crecimiento económico y la protección ambiental. Reafirmándose el carácter estructural y horizontal de la Política Ambiental.

El Acta Única pretende desarrollar una nueva estrategia, tanto jurídica como política en materia de medio ambiente, de manera que las consideraciones ecológicas se integren realmente en las decisiones futuras sobre planificación y desarrollo de todos los aspectos de la vida económica y social de la Comunidad Económica Europea.

El carácter preventivo de esta Política se ve potenciado por la «Directiva del Consejo 85/337/CEE, de Evaluación de Impacto Ambiental». Por ser una Directiva permite la adecuación del Derecho Comunitario al de los Estados miembros pero que, obliga a someter al procedimiento de evaluación, a aquellos proyectos y actividades públicas o privadas que puedan tener una incidencia notable sobre el medio ambiente en razón de su naturaleza, dimensión o localización.

Aparte de las líneas de actuación ya enunciadas por otros documentos comunitarios, se trata de favorecer una mayor atención e integración de la política del medio ambiente en las distintas políticas de la Comunidad y de sus Estados miembros (políticas económica, industrial, agrícola y social).

$Y$, es que como afirma LóPEZ, A. (1990): «El significado supone un giro de casi 360 grados en tanto que las políticas sectorial (muchas de ellas causantes de impactos ambientales) no solamente tendrán, a partir de ahora, la necesaria componente medioambiental, sino que sobre todo, tendrán que converger en la política de medio ambiente como matriz de interconexión y como fuente y objetivos en vistas a alcanzar la calidad de vida de los ciudadanos europeos. La Directiva comunitaria sobre Evaluación de Impacto Ambiental no solamente tiene su correlato de precauciones y de controles de toda obra pública y privada, sino que además, el medio ambiente ha encontrado una sanción tan favorable como de exigencia para el resto de las políticas...". 
Existe, por lo tanto, una interdependencia entre medio ambiente y desarrollo, política regional y política de medio ambiente.

Por último, se establecen unos umbrales mínimos, que por supuesto cada Estado miembro puede incrementar para alcanzar una mayor calidad de vida.

El Cuarto Programa de Acción (1987-1992) ${ }^{5}$. Supone la consolidación de los objetivos de los primeros programas de acción y además, de los del Año Europeo de Medio Ambiente y del Acta Única Europea. Así, en la Resolución del Consejo de 16 de diciembre de 1986 relativa al fortalecimiento de la acción comunitaria en favor del medio ambiente se afirma:

“...dicho Programa ofrece una oportunidad para fortalecer de manera decisiva, a partir de los resultados logrados en el pasado, la acción comunitaria en ese ámbito y para establecer un marco coherente dentro del cual se pueden formular, coordinar y ejecutar acciones comunitarias específicas durante el período 1987 a 1992».

Los problemas permanentes $-y$, en muchos casos, crecientes- del deterioro del medio ambiente han convencido a la Comisión de que la adopción de normas estrictas para la protección del medio ambiente no es únicamente una mera opción, se ha convertido en algo esencial. Por otra parte, la Comisión también considera que dada la demanda pública creciente de normas perfeccionadas de protección del medio ambiente y de artículos no perjudiciales para el mismo, la industria comunitaria sólo será viable si se adapta gradualmente a los requisitos de tales normas y a la producción de dichos artículos. Así, un alto nivel de protección del medio ambiente se ha convertido en un imperativo de carácter económico.

En este sentido, el propio Programa indica que si bien es cierto que una política de medio ambiente correcta sólo es posible si se progresa al mismo tiempo en el campo económico y social, también es cierto que el progreso económico y social duradero sólo es posible si los aspectos medioambientales se tienen en cuenta como un elemento esencial del desarrollo económico y social.

$Y$, es que, aunque los beneficios económicos derivados de las medidas de medio ambiente sólo pueden obtenerse a largo plazo, existen otras

\footnotetext{
5 Aprobado por Resolución del Consejo de las Comunidades Europeas y de los Representantes de los Gobiernos de los Estados Miembros reunidos en el seno del Consejo de 19 de octubre de 1987, relativa a la continuación y aplicación de una política y de un programa de acción de las Comunidades Europeas en materia de medio ambiente (1987-1992).
} 
razones medioambientales y económicas importantes para realizar las inversiones necesarias. La OCDE ha manifestado que «...los beneficios que se derivan de las medidas de medio ambiente (incluidos los costes por daños evitados) han sido, en general, superiores a sus costes". Por ello, es fundamental, calcular y disponer, con fines comparativos, de los costes que se producen como consecuencia de no realizar una acción de medio ambiente.

La Comisión se encarga de velar por que se adopten las medidas para integrar los requisitos de protección del medio ambiente en la planificación y la ejecución de las políticas económica, industrial, regional, transportes, energía,..., agrícola y social.

En este sentido, las áreas urbanas tienen un tratamiento primordial dentro del Cuarto Programa. La urbanización rápida resultante de la migración del campo a la ciudad y el crecimiento actual de la propia población urbana, trae como consecuencia viviendas insuficientes y deterioro de la calidad del medio ambiente, procesos que se han visto acompañados de procesos de suburbanización. La población y las inversiones recientes han buscado lugares distantes de las antiguas con urbanizaciones industriales que se han visto afectadas negativamente por los cambios estructurales de la economía que han producido altos niveles de desempleo, lugares abandonados o contaminados, edificios vacios y un conjunto de viviendas e infraestructuras obsoletas. Incluso, se puede afirmar que las condiciones de vida en algunas de estas aglomeraciones ha descendido notablemente.

Por ello, es primordial la resolución de los problemas de medio ambiente urbano. La acción necesaria debe incluir: el aprovechamiento de tierras abandonadas o contaminadas; la creación de parques y otras zonas de paisaje (sistema de espacios libres); el tratamiento de los lugares antiestéticos y medidas de restauración de edificios antiguos: rehabilitación urbana; control del crecimiento urbano.

En definitiva, se trata de promover, de elevar la calidad de vida en el medio urbano, fomentar la economía local, y a su vez, sentar las bases de la renovación económica.

La Comisión se encarga de analizar las formas posibles de colaboración entre los sectores público y privado y otros intereses para lograr la rehabilitación de determinadas zonas urbanas, contribuyendo a su desarrollo económico. $Y$, a través de los fondos estructurales comunitarios (especialmente el Fondo Regional Europeo) abordar más eficazmente los problemas de las zonas urbanas y garantizar un nivel adecuado de financiación que permita a la Comunidad participar, junto con los otros 
sectores, en los planes de renovación urbana, integrando los principios de protección del medio ambiente y los de la política regional.

Las zonas montañosas y costeras son de naturaleza similar en cuanto a los problemas a que se enfrentan, deben desempeñar funciones en conflicto. Deben garantizar el bienestar de la población local, alojar a un número creciente de turistas de todas las zonas de Europa y, al mismo tiempo, proteger los medios. Por ello, el Cuarto Programa centra sus esfuerzos tanto en la Carta Costera como en adaptar la Directiva 268/75/ CEE sobre agricultura de montaña y de determinadas zonas desfavorecidas, para contribuir de forma más eficaz al mantenimiento de los medios ambientes valiosos y, al mismo tiempo, de los ingresos de los ciudadanos.

\section{II.2. La Directiva del Consejo 85/337/CEE relativa a la evaluación de las repercusiones de determinados proyectos públicos y privados sobre el medio ambiente}

A la vista de los Tratados y de los Programas de Acción la mejor política del medio ambiente consiste en evitar, desde su origen, la creación de contaminación o de perjuicios más que de combatir ulteriormente sus efectos; de ahí la necesidad de tener en cuenta los impactos posibles sobre el medio ambiente de todos los procesos técnicos de planificación $y$ de decisión.

Desde el Primer Programa Comunitario (1973-1976) se sostiene que conviene tener en cuenta en todo lo posible la incidencia de todos los procesos de planificación y de decisión sobre el medio ambiente, que no puede ser considerado como un medio externo al hombre, sino que más bien debe ser considerado como un dato indisociable de la organización y de la promoción del progreso humano y que por tanto puede sufrir atentados y agresiones. Debido a ello conviene evaluar las consecuencias que sobre la calidad de vida y el medio natural, tiene toda actuación de tipo territorial realizada, o por realizar, a nivel nacional o comunitario.

El procedimiento que asegura la toma en consideración de los daños que pueda sufrir el medio ambiente dentro de cualquier proceso, es la evaluación de impacto ambiental. Por ello, debe ser introducida de forma progresiva en todas las actividades humanas susceptibles de causar daños y perjuicios al medio ambiente, incluyendo en este grupo: obras públicas o privadas, los planes de ordenación del territorio, los programas económicos y de desarrollo regional, nuevas tecnologías, actos jurídicos, etc.

No obstante, como luego se comprobará, un hándicap importante para su puesta en práctica lo constituyen las desigualdades entre las diversas 
legislaciones vigentes en los diferentes Estados miembros en materia de repercusiones sobre el medio ambiente.

Los trabajos para la elaboración de la directiva 337/85/CEE se iniciaron por una propuesta de la Comisión al Consejo el 16 de junio de 1980. La notificación a los Estados miembros tuvo lugar el 3 de julio de 1985, fecha a partir de la cual disponian de tres años para su adopción.

La directiva 337/85/CEE se compone de 14 artículos y 3 anexos, que deben contribuir a evaluar los efectos que un proyecto sobre el medio ambiente puede provocar, para proteger la salud humana, contribuir mediante un mejor entorno a aumentar la calidad de vida, velar por el mantenimiento de la diversidad de especies y conservar la capacidad de reproducción del ecosistema como recurso fundamental de la vida.

En su texto se determinan los proyectos públicos o privados susceptibles de tener una incidencia en el medio ambiente, aunque del mismo se excluyen los referidos a fines de defensa nacional (estratégicos) y los proyectos aprobados a través de un acto legislativo nacional específico. A la vez se regulan y disponen las obligaciones que competen a todos los Estados miembros para integrar las EIA en los procesos de autorización o concesión. También se señala que las EIA deben evaluar las repercusiones, identificarlas, describirlas en función de cada caso en particular.

Especial interés presenta el artículo 13, que introduce la posibilidad de aplicar normas más estrictas, textualmente señala: «La presente Directiva no afectará a la facultad que tienen los Estados miembros de establecer normas más severas en lo que se refiere al campo de aplicación y al procedimiento en materia de evaluación de las repercusiones sobre el medio ambiente».

En la regulación se deslinda entre los proyectos que tienen obligación de atenerse a una evaluación sistemática, y aquellos que se someterán a una evaluación cuando los Estados consideren que sus características así lo requieran. Entre los últimos se contemplan los proyectos que no implican una evaluación sistemática pero en los que sería recomendable su aplicación. En este grupo es donde se incluyen los vinculados con la planificación y ordenación territorial. En consecuencia, dado que el artículo 13 de la directiva permite el establecimiento de normas más estrictas, las propuestas constituyen recomendaciones que han sido recogidas en las diversas legislaciones de los Estados miembros de la CEE.

\section{EXPERIENCIAS EN LAं APLICACIÓN DE LA EVALUACIÓN DE IMPACTO AMBIENTAL}

Como se ha expuesto en el apartado anterior, las EIAs han sido consideradas por la Comunidad Europea como el instrumento más adecuado 
para la conservación y preservación de los recursos naturales y la defensa del medio ambiente (LóPEz BUSTOS, F.L. 1991).

La Directiva que regula la EIA contempla la forma y amplitud en que ésta se debe realizar: proyectos que, en cualquier modo, deben ser sometidos a una EIA; proyectos en los que se recomienda la EIA, dependiendo de las características de cada Estado; por último, se señala la información que debe aportar el responsable del proyecto.

En el proceso de aplicación de las EIA se han despertado algunos interrogantes sobre su estrecho campo de aplicación. Tales interrogantes se dirigen hacia la extensión de la EIA dentro del proceso de planeamiento. Así cabe cuestionarse isi las EIA deben aplicarse a políticas, planes y programas y no, tan sólo, a proyectos?

La reflexión en relación a la ampliación de la EIA a otras categorías superiores del planeamiento se ha extendido entre técnicos y estudiosos de la evaluación ambiental de todo el mundo.

Se indica desde esta nueva concepción que el planeamiento es un proceso escalonado en el que el proyecto es la fase final, que se ve antecedida por políticas - nivel superior-planes y programas. La política «...puede considerarse como la inspiración y guía para la acción, el plan como un conjunto de objetivos coordinados y ajustados en el tiempo para el cumplimiento de la política, y el programa como un conjunto de proyectos en un área particular..." (WOOD, C.; LEE, N.; WALSH, F.; JONES, C.E. 1990, 2-3).

Los informes de EIA son susceptibles de aplicarse a políticas, programas y planes, aunque su índice y objetivos puedan ser modificados. Incluso ciertos impactos ambientales no son evaluables a nivel de proyectos y si acudiendo a otras categorías superiores. Diferentes tipos de impactos pueden ser evaluados en diferentes etapas.

La ampliación de la EIA puede dirigirse hacia diversos sectores de la actividad económica, así como a planes de ocupación y apropiación de suelo. En tal sentido, puede aplicarse desde una escala nacional, y también regional o local, hasta a acciones sectoriales y de planificación del medio físico.

\section{III.1. Antecedentes en la aplicación de EIA a categorías superiores a proyectos}

III.1.1. El ejemplo californiano en la aplicación de la EIA

En EE.UU. la experiencia en evaluación ambiental se puede establecer desde el 1 de enero de 1970, fecha en la que entra en vigor la "National 
Environmental Policy Act” (NEPA), a través de la que se decide, según procedimientos ambientales, la aprobación de proyectos y programas.

El procedimiento de «Environmental Impact Statement» (EIS) trata de incorporar a la toma de decisión los aspectos ambientales del proyecto o programa en cuestión con sus implicaciones a corto y largo plazo, directos e indirectos, incorporando el impacto sobre el hombre, su entorno físico y social y, la naturaleza (ALLende LANDA, J. 1990).

La aplicación a programas y políticas de estas medidas aumenta el carácter preventivo de las evaluaciones al minimizar el alcance de las decisiones propuestas. En esta línea se incide también en la identificación y evaluación de forma sistemática de las posibles alternativas.

En el mismo plano se relaciona el procedimiento de evaluación ambiental con la ordenación del territorio, identificando conflictos de los planes, a diversas escalas, de usos del suelo.

Dentro del ámbito estadounidense destaca la aplicación realizada en California de las EIAs a las políticas, planes y programas.

La Ley de Calidad Ambiental de California (California Environmental Quality Act -CEQA) obliga a realizar una EIA a proyectos, pero de la misma forma a políticas, planes y programas. Estos últimos informes realizados a políticas, planes o programas se denominan IIA (Programme "EIRs»).

En California las instituciones públicas deben elaborar IIA de programas por las Directrices de la Ley de Calidad Ambiental del Estado de California (State CEQA Guidelines) - reglamento de la Ley de Calidad Ambiental-.

Es obligatorio realizar un IIA para los programas con las siguientes implicaciones:

- actividades con conexión geográfica,

- actividades que forma parte de una cadena,

- leyes, reglamentos o planes que rigen la marcha de un programa continuado de actividades,

- acciones individules dirigidas por una sola autoridad reguladora, con efectos ambientales similares y que pueden corregirse por medios también similares.

De la utilización de las Directrices de CEQA se derivan cinco ventajas ambientales:

- dan ocasión de considerar de forma más exhaustiva impactos y alternativas que en el caso de utilizar un IIA sobre una actividad especifica, 
- ponen de manifiesto impactos ambientales acumulados,

- evitan a la Administración Pública una reconsideración permanente y parcial de políticas,

- permiten considerar alternativas a políticas y aplicar éstas de forma flexible,

- reducen los procedimientos administrativo-burocráticos al considerar fuentes de información múltiples e interrelacionadas.

El uso más común de IIA en California ha sido en relación a planes generales municipales 0 de comunidades.

Las diferencias en una evaluación ambiental referida a un plan o programa y un IIA de proyectos se establece en la amplitud y generalización, mayor en el caso del programa. Las IIA tienen una utilidad más amplia cuando analizan los impactos ambientales de un plan o programa de manera tanto específica como de conjunto. Incluso ello puede evitar en algún caso el desarrollo de IIA de proyectos, cuando se han evaluado sus efectos derivados en el IIA de programa. Ello está relacionado con la "metodología escalonada" que parte de las categorías más amplias para descender a otras más concretas.

En la experiencia de aplicación de IIAs en California se han advertido dos dificultades que es importante reseñar aquí. En especial la falta de uniformidad de los criterios empleados en las IIAs y en la formación adicional necesaria para la preparación de IIAs de programas; y, en relación a la metodología a seguir para abordar estudios de IIAs con una calidad mínima.

\section{III.1.2. Planeamiento integrado y evaluación ambiental en Holanda}

En Holanda a lo largo del presente siglo se ha desarrollado un proceso de urbanización que se ha manifestado en una mayor diferenciación funcional de las ciudades (centro-periferia), y en la aparición de fenómenos de suburbanización, básicamente de dos tipos: a) transformación de núcleos agrarios o postagrarios en núcleos industriales o suburbios residenciales; b) transformación de ciudades ya existentes en nuevas ciudades dormitorios dependientes de las grandes aglomeraciones.

Estas transformaciones - acelerado proceso de urbanización- en el espacio hicieron necesaria una modificación de la política territorial, ante el grave deterioro que se estaba produciendo en el medio ambiente, traducido en la pérdida del paisaje más tradicional de Holanda.

Hasta 1960 el planeamiento desarrollado se hacía sobre la base fundamentalmente municipal. En 1950 una comisión constituida para exa- 
minar la normativa territorial existente preconiza la separación entre la legislación del espacio y la legislación referente a la habitación, que es tan sólo uno de sus aspectos. Fruto de estas nuevas directrices en 1956 se redactó un proyecto de ley, que finalmente no fructificó, en el que se establecía el problema de la ordenación del espacio a tres niveles: físico, económico y social.

A partir de 1960 la planificación adquiere un carácter global. Así, en 1958, como continuación al de 1956, se realizó un proyecto de ordenación territorial de carácter nacional, bajo los siguientes principios: definición del uso del suelo según las actividades económicas; defensa contra el agua y protección de la naturaleza. En 1965, se establece el marco jurídico para la intervención del Estado en la ordenación del espacio de Holanda. La política emanada de estos nuevos planteamientos territoriales se denomina «desconcentración-con centrada" y, significa la creación de un espacio urbano reestructurado con el fin de restaurar las funciones urbanas, es decir, urbanización más compacta, usando las infraestructuras existentes, racionalizando el consumo de espacio y recursos y, por último, estimulando la economía en consonancia con las demandas de los ciudadanos.

No obstante, según el informe «Third Report on Physical Planning» realizado en 1976, se recoge la preocupación de la Administración por los procesos de suburbanización, el aumento de los movimientos pendulares, y el deterioro de los espacios abiertos. Este documento supone un elemento clave en la política ambiental holandesa, ya que pone de manifiesto como la preocupación por los valores ecológicos se sitúa en un primer plano. Ello se traduce en un incremento de los recursos dedicados a investigación ambiental y la preocupación por crear nuevas áreas naturales. Todo ello desde la colaboración entre científicos y técnicos, puesto que el planeamiento ambiental no se concibe de forma cerrada, sino en continuo proceso de revisión, en función de los cambios que se producen en las necesidades para alcanzar un mayor nivel y calidad de vida (Paniagua Mazorra, A.; Tarancón Rico, O.P. 1990).

Fruto de este largo proceso de maduración, la ElA se introduce en Holanda durante 1987. La EIA se utiliza como proceso de integración de la política ambiental: integración de la planificación y toma de decisiones de organizaciones públicas y privadas, en este sentido se produce una integración externa con instancias para las cuales los problemas ambientales no son prioritarios en su actuación.

En otro plano hay que considerar las diversas políticas ambientales para las que se intenta lograr una integración interna (integración de políticas sobre el agua, suelo, atmósfera, etc.), se realiza mediante el Plan 
Nacional de Política Ambiental (NEPP), publicado desde mayo de 1989, regulación a la que se añaden nuevas medidas en 1990.

La NEPP define la estrategia ambiental del conjunto del Estado. Su aplicación se produce en los siguientes sectores: agricultura, industria, transporte, energía, construcción y gestión de recursos. En relación a cada sector se realiza una adaptación de la NEPP, que supone su integración externa con políticas de orden superior.

De esta manera, la EIA se utiliza como una herramienta de apoyo en la toma de decisiones concretas y la NEPP como un instrumento para la formulación de políticas. Asimismo, existe un proceso de influencia de la EIA en los procesos de toma de decisiones.

En conjunto, se plantea una gestión integrada que abarque el ciclo completo recursos-producción-residuos, es decir, la aplicación de la EIA en combinación con la planificación para lograr su optimización.

\subsection{El desarrollo de la EIA en la CEE}

Durante los dos últimos años los diversos países de la CEE han dirigido sus esfuerzos a adaptar la legislación de cada Estado a la Directiva de EIA comunitaria ${ }^{6}$. También se han ocupado de preparar directrices técnicas y de procedimiento. Incluso los estados con mayor tradición en la materia han potenciado sus propios estudios de evaluación (Francia, Holanda y Gran Bretaña).

Desde la propia CEE se han realizado actividades para ampliar y fortalecer la Directiva 85/337, en especial con el fin de ampliar la lista de proyectos agrícolas que deben someterse a EIA y la extensión de la EIA a ciertas políticas, planes y programas.

En cada país se ha presentado problemas particulares, y la adaptación a la normativa comunitaria, también se ha realizado de manera diferente.

En los Países Bajos destaca el modelo holandés, en el que se ha inspirado el belga.

El decreto de evaluación de impacto ambiental de Holanda se aplica desde el 1 de septiembre de 1987. En el mismo se contemplan 27 actividades sometidas a evaluación ambiental (con diversas subcategorías). En conjunto, hasta mediados de 1990 se han iniciado ElA de 189 activi-

- La documentación utilizada en este epigrafe se debe al Centre for EIA de Manchester. 
dades. En su mayor parte se refieren a tratamiento de residuos $(47,1 \%)$, principalmente vertederos; y a instalación de zonas de recreo al aire libre (sobre todo campos de golf) $(15,9 \%)$.

El ministerio holandés de Urbanismo, Planificación Física y Medio Ambiente, así como el Ministerio de Agricultura, Naturaleza y Pesca pueden conceder exenciones de realizar un EIA incluso a proyectos contemplados en los anexos de la directiva 85/337/CEE.

El hecho que en Holanda no se hayan realizado excesivas EIA sobre tratamiento de agua, recuperación de tierras, se debe a la existencia de un fuerte organismo público que controla todo este tipo de actividades de gran tradición en este país.

En Bélgica la EIA, que se inspira en el modelo holandés, es responsabilidad de los gobiernos regionales (Flandes, Valonia y Bruselas).

Las principales diferencias respecto al modelo holandés son las siguientes:

- Ios umbrales a partir de los que entran en vigor las leyes sobre EIA en relación a instalaciones industriales son más bajos en Bélgica, lo que conlleva un número mayor de instalaciones sujetas a EIA,

- en la legislación belga se exige la intervención de expertos independientes en la elaboración del estudio en algunas de las disciplinas básicas contempladas en la directiva $85 / 337$. En cambio en la reglamentación holandesa se precisa su intervención en la preparación de directrices específicas acerca del alcance y contenido del estudio, así como en el control de calidad del informe emitido,

- la información pública en todas las fases de EIA es mucho mayor en Holanda,

- por último, el tratamiento de alternativas es mucho más exigente en la reglamentación holandesa.

Por su parte, en Luxemburgo la reglamentación de EIA está en fase de proyecto, aunque muy avanzada, en el que se contemplan dos tipos de regulación sobre EIA: una primera concretará los proyectos que deben someterse a EIA, mientras que otra cubrirá el procedimiento de información pública y la publicación de decisiones administrativas.

Se prononen, asimismo, dos categorias de proyectos y, por tanto, dos tipos de EIAs.

Los proyectos contemplados en el anexo 1 de la directiva 85/337/CEE, además de proyectos de consolidación de suelos estarán sometidos a una EIA completa. Se recomienda la inclusión de los costes de cualquier medida correctora sugerida. Otros tipos de proyectos sólo tienen que so- 
meterse a una EIA simplificada, denominada «noticia de impacto» (Projet de Loi n. 3257/20-9-1988).

En general, se contempla en todas las fases de EIA y EIA simplificada la información pública.

En Dinamarca todos los proyectos contemplados en la directiva 85/ 337, anexos 1 y 2 debían de someterse a una EIA desde la década de 1970. Con la normativa comunitaria se amplía la legislación nacional, para incluir los proyectos estipulados en el anexo 3 de la directiva CEE.

Las ElAs de proyectos se consideran suplementos del plan regional aprobado. Tales suplementos se desarrollan de acuerdo con los procedimientos ya existentes de planificación regional. Finalmente cada EIA y proyecto tiene que ser aprobado por el Ministerio de Medio Ambiente ${ }^{7}$.

En Alemania como en Dinamarca también se ha tratado de integrar la EIA en la legislación y procedimientos previos. La Ley de Evaluación de Impacto Ambiental conocida como "UUP Gesetz", entró en vigor el 1 de agosto de 1990. Esta ley modifica 11 previas tendrán que incluir una cláusula relativa a EIA.

Solamente tienen obligación de realizar EIA los proyectos. Los planes y programas no están sujetos a la EIA. No obstante, los planes de usos de suelo o los cambios que se deseen introducir en planes regionales con el fin de obtener una licencia o permiso para un proyecto sí están sometidos a EIA. También es obligatoria la EIA en el suelo calificado de urbanizable, aunque no se haya programado.

7 Los siguientes proyectos están sometidos a EIA:

- refinerías de petróleo y grandes instalaciones de gasificación y licuefacción de carbón,

- grandes centrales térmicas y centrales nucleares,

- instalaciones dirigidas al almacenamiento de residuos radioactivos,

- trabajos integrados de fundición de hierro colado y acero,

- instalaciones químicas integradas,

- construcción de autopistas, autovías y carreteras generales,

- aeropuertos,

- grandes puertos,

- instalaciones para la ubicación de residuos,

- instalaciones químicas incluidas en la directiva de Seveso de la CEE,

- puesta en cultivo de áreas naturales de más de 300 has.,

- drenaje y puesta en regadio de áreas de más de 300 has.,

- minería a cielo abierto de más de 10 años o con una producción anual superior a 200.000 $\mathrm{m}^{3}$,

- instalaciones dedicadas a fabricación de cemento y arcillas,

- explotaciones de caliza con producción anual mayor de $200.000 \mathrm{~m}^{3}$,

- instalaciones hoteleras de más de 75 habitaciones situadas en áreas costeras o de especial interés natural. 
En Gran Bretaña se ha implementado de forma suave la reglamentación de EIA, en todo caso los procedimientos han sido sometidos a numerosas consultas, lo que ha conducido al gobierno a realizar un memorándum sobre medio ambiente (White Paper) publicado en otoño de 1990. Se pretende mejorar la preparación y evaluación de los estudios de impacto ambiental, aunque sin establecer procedimientos rígidos. La ampliación del EIA al planeamiento aunque ha recibido consideración favorable, de momento no se ha desarrollado.

En Irlanda también el desarrollo de la directiva comunitaria se ha realizado de forma compleja, ya que ha dado lugar a cuatro reglamentaciones. Se ha optado por el procedimiento de establecer umbrales a diversos proyectos $^{8}$.

Los ayuntamientos puede exigir a su discrección una EIA a proyectos a realizar en su término municipal con umbrales inferiores.

Los países del sur de la CEE - a excepción de España al que se hará referencia más adelante - han aplicado la Directiva de EIA de una forma incierta e inconscientemente desde la propia adaptación de la directiva a la legislación nacional, a su aplicación por promotores y técnicos, pasando por diversas barreras sociales y administrativas.

En Grecia se han considerado dos tipos de ElA, las "normales" y las EIAs "especiales", relativas a lugares de particular belleza natural.

En Italia es donde la aplicación de la directiva tiene mayores dificultades ya que no anula ningún procedimiento previo. Por su parte, en Portugal los problemas han surgido por un uso deficiente e inapropiado de métodos y técnicas de EIA.

\section{LA EVALUACIÓN DE IMPACTO AMBIENTAL EN ESPAÑA}

El Real Decreto 1302/1986 de 28 de junio, de Evaluación de Impacto Ambiental, publicado en el Boletín Oficial del Estado núm. 155 del 30 de

- Los proyectos con umbrales asociados que precisan EIA son:

- desarrollo de áreas industriales con una superifice superior a 15 has.,

- proyectos de desarrollo urbano que abarquen una superficie mayor de 50 has. en el caso de nuevas áreas urbanas o de expansión de áreas urbanas ya existentes, y una superficie superior a 2 has. si es en zona urbana ya existente,

- plantas depuradoras con una capacidad para una población superior a 10.000 hab.,

- oleoductos o gaseoductos con una longitud mayor de $80 \mathrm{~km}$.,

- cualquier fábrica de aceite o harina de pescado,

- todas las extracciones de petróleo.

- todas las instalaciones de producción de cemento. 
junio de 1986, constituye la adaptación al ordenamiento interno español de la directiva 85/337/CEE de 27 de junio de 1985, dentro del período establecido por la CEE.

Anteriormente a este Real Decreto aparecen de manera incipiente conceptos y denominaciones en la legislación española, porque el medio ambiente es un bien jurídico, de interés general y patrimonio colectivo, que debe ser titulado jurídicamente. Se trata de diversas disposiciones con diferentes rangos jurídicos, que se referían a la necesidad de evaluar los efectos ambientales de determinados proyectos 0 actividades. Sin embargo, son normas sectoriales, que debido a su descoordinación, establecian regulaciones de carácter fragmentario y disperso, por lo que recibian un tratamiento marginal dentro de las propias disposiciones ${ }^{9}$.

EI R.D. de E.I.A. completa y normaliza este procedimiento administrativo, como instrumento más adecuado para la preservación de los recursos naturales y la defensa del medio ambiente, mediante la aplicación de una técnica, que se encuentra normalizada en otros países industrializados y recomendada por el PNEUMA, OCDE y CEE, que introduce la variable ambiental en la toma de decisiones sobre los proyectos con incidencia importante en el medio ambiente.

La Evaluación de Impacto Ambiental es un proceso de doble carácter. Trata de elaborar un análisis encaminado a predecir las alteraciones que un proyecto $O$ actividad puede producir en la salud humana y en el medio ambiente. Por el otro, establece el procedimiento jurídico-administrativo para la aprobación, modificación o rechazo de la actividad o proyecto por parte de la Administración.

La finalidad es dotar a los responsables de la planificación y gestión ambiental de una herramienta que les permita asegurarse de qué decisiones transcedentales, en el campo del medio ambiente, se han tomado

9 Las principales disposiciones son:

1. Reglamento de actividades clasificadas, de 30 de noviembre de 1961 , que regula sus repercusiones sobre la sanidad ambiental y propone sistemas de corrección.

2. Decreto $833 / 75$, de 6 de febrero y orden del Ministerio de Industria de 18 de octubre de 1976, sobre protección del medio ambiente atmosférico y en relación con los proyectos de nuevas industrias potencialmente contaminadoras de la atmósfera y ampliación de las existentes.

3. Ley de Minas de 21 de julio de 1973 y reales decretos de 15 de octubre de 1982 y 9 de mayo de 1984, sobre actividades mineras, especialmente en lo que se refiere a planes de res. tauración del espacio natural afectado por extracciones a cielo abierto.

4. Ley de Aguas de 2 de agosto de 1985 y R.D. 849/86, de 22 de abril (Reglamentando varios de sus Títulos), en relación con la protección ambiental del dominio público hidráulico y de las aguas continentales. 
después de un análisis científico, sistemático y adecuado. En definitiva, proporcionar mayor fiabilidad y confianza a las decisiones que deben adoptarse, para poder elegir, entre diferentes alternativas posibles aquella que mejor salvaguarde los intereses generales desde una perspectiva global e integrada y teniendo en cuenta todos los efectos derivados de la actividad proyectada.

Respetando los mínimos establecidos en el anexo I de la directiva comunitaria, incluye otras actividades que deben ser objeto de EIA, de entre las comprendidas en el anexo II, que cada Estado miembro puede incorporar, según su criterio, a este procedimiento.

Contiene Disposiciones Adicionales primera y segunda que representan importantes limitaciones para la EIA y que según LóPEZ Bustos (1991) «parece bastante excesivo». No será de aplicación a los proyectos relacionados con la defensa nacional, ni con los aprobados específicamente por una Ley del Estado, ni tampoco a los supuestos excepcionales en los que el Consejo de Ministros mediante acuerdo motivado, pueda excluir a un proyecto determinado del trámite de evaluación de impacto. Este último caso, precisa que el acuerdo del Gobierno sea público y contendrá las previsiones que en cada supuesto estime necesarias en orden a minimizar el impacto ambiental del proyecto.

EI R.D. recoge las dificultades existentes para la definición de una normativa en el campo ambiental, utiliza cláusulas generales, conceptos indeterminados, etc. El estudio de impacto obedece a un método científico que puede resumirse en la definición del problema, formulación de las hipótesis, valoración de las mismas, elección de la menos negativa, previsión de sus resultados, incorporación de correctores y verificación de si los resultados son conformes o no con las previsiones. En suma, las evaluaciones de impacto ambiental, informan $y$, a la vez, dan apoyo o justifican las decisiones administrativas, tanto sobre actividades públicas como privadas.

Las EIA cobran sentido dentro de un procedimiento administrativo de toma de decisiones y ellas mismas requieren un procedimiento especial, más o menos independiente, según el valor $u$ obligatoriedad que se de a la evaluación o declaración (FUenTES BODELón 1984, pág. 539).

El Real Decreto 1131/1988, publicado en el Boletín Oficial del Estado de 5 de octubre de 1988, aprueba el Reglamento para la ejecución del R.D.L. de Evaluación de Impacto Ambiental. En él se desarrolla la normativa y en dos anexos se definen una serie de conceptos técnicos relacionados con la EIA y, se precisan las características y condiciones de los proyectos sometidos obligatoriamente al procedimiento de evaluación. 
El R.D.L. tiene carácter de legislación básica, según se estipula en su artículo 1; el Reglamento, como desarrollo de la normativa básica, es directamente aplicable a la Administración Central del Estado y a las Comunidades Autónomas que carezcan de competencia legislativa en materia de medio ambiente, y supletoriamente si la tienen atribuida en sus Estatutos de Autonomía (Art. Único del R.D. 1131/88).

El preámbulo indica que la Evaluación de Impacto Ambiental, «lejos de ser un freno al desarrollo y al progreso, supone y garantiza una visión más completa e integrada de las actuaciones sobre el medio en que vivimos, una mayor creatividad e ingenio, mayor responsabilidad social en los proyectos, la motivación para investigar en nuevas soluciones tecnológicas y, en definitiva, una mayor reflexión en los procesos de planificación y toma de decisiones».

El Reglamento recoge la problemática especial de los proyectos con efectos transfronterizos.

Por último, se incluyen dos anexos. En el primero se establecen los conceptos básicos de proyecto, titular de proyecto o promotor, etc. ${ }^{10}$.

En el segundo se definen las especificaciones relativas a las obras, instalaciones o actividades comprendidas en el anexo del Real Decreto Legislativo 1302/1986, de 28 de junio, de evaluación de impacto ambiental. En él se incluyen los recogidos en el ANEXO I de la directiva comunitaria 85/337/CEE y se añaden otros pertenecientes al segundo ANEXO II, que constituyen recomendaciones; entre los pertenecientes al segundo caben citar las repoblaciones forestales, la construcción de presas, ..., en cambio, no relaciona los proyectos de ordenación rural, los trabajos de ordenación de zonas industriales, trabajos de ordenación urbana, ni los de equipamientos turísticos, como complejos hoteleros, etc.

A través del análisis del Reglamento se advierte que en el caso español la trasposición de la directiva comunitaria no se ha incluido la planificación territorial, a pesar de las recomendaciones constantes de los Programas de Actuación de la CEE y, especialmente, del IV Programa.

\footnotetext{
10 Los aspectos precisados en el primer anexo son los siguientes: conceptos básicos del proyecto, titular del proyecto o promotor, autoridad competente sustantiva, autoridad competente de medio ambiente, estudio de impacto ambiental, declaración de impacto, efecto notable, efecto mínimo, efecto positivo, efecto negativo, efecto directo, efecto indirecto o secundario, efecto simple, efecto acumulativo, efecto sinérgico, efecto a corto, medio o largo plazo, efecto permanente, efecto temporal, efecto reversible, efecto irreversible, efecto recuperable, efecto irrecuperable, efecto periódico, efecto de aparición irregular, efecto continuo, efecto discontinuo, impacto ambiental compatible, impacto ambiental moderado, impacto ambiental severo e impacto ambiental crítico, que contribuyen a clarificar el vocabulario ambiental del reglamento.
} 
Tampoco se han recogido las recomendaciones dadas por Naciones Unidas (PNUMA), relacionando el desarrollo sostenido y la ordenación racional del medio, en la etapa inicial de planificación y la adopción de las evaluaciones de impacto ambiental. Así el primer objetivo es: promover la adopción y aplicación por parte de los Estados de mecanismos apropiados tanto jurídicos como de orden para evaluar los efectos sobre el medio ambiente de actividades potencialmente nocivas, bajo su jurisdicción y control, así como la divulgación de información y uso de éste por el público. Promover el uso de la evaluación de los efectos sobre el medio ambiente (sean nacionales o internacionales) como elemento esencial de la planificación del desarrollo. Tampoco se adoptan las recomendaciones dadas por la OCDE sobre la utilización de la técnica de evaluación de impacto ambiental para elevar la calidad de vida de todos los ciudadanos.

En 1984, la Directora General del Medio Ambiente, doña Concepción Saenz Laín (Curso 1984), afirmaba en el prólogo de un curso de impacto ambiental:

«Incorporadas ya a la normativa de la mayoría de los países avanzados, los EIA tienden a ser un componente obligatorio de los procesos de planificación y de desarrollo."

En defintiva, la EIA puede ser también un instrumento de planificación y de gestión integrada de los recursos naturales y puede contribuir de manera importante a asegurar el desarrollo equilibrado y sostenido de las actividades económicas y las condiciones de vida, es decir, procurando el desarrollo económico y social y al tiempo la conservación y mejora del medio ambiente en orden de elevar la calidad de vida y la utilización racional de los recursos, tal y como propugna el artículo 45 de la CEE. Pero, en el actual marco de la legislación española ha quedado excluida su aplicación a la ordenación del territorio.

\section{IV.1. Aplicación de la EIA en España}

La aplicación en España de la directiva 85/337/CEE ha puesto de manifiesto diversos inconvenientes de su adaptación al ámbito nacional.

La EIA muestra una dependencia del proyecto en cuestión, y de las fases del mismo. Concretamente en carreteras y presas la EIA se realiza respectivamente sobre el estudio informativo y el anteproyecto, por lo que no abarca todas las fases del proyecto, ni la localización definitiva, asi como la viabilidad socioeconómica. 
Por otra parte la calidad de los análisis realizados se puede calificar de baja, al presentar diferentes carencias y falta de concreción. Este rasgo es similar al del resto de países mediterráneos de la CEE, en los que no ha existido una tradición de estudios ambientales.

Se ha indicado desde la Secretaría General de Medio Ambiente que «...la información disponible (en las ElAs) suele ser en la mayoría de los casos poco ajustada al caso específico de que se trata..." (MEDIo 1991, 76). Ello pese a que el Reglamento de ElA establece que cada estudio debe adaptarse al proyecto en concreto que se evalúa. Este hecho repercute negativamente sobre las Declaraciones de Impacto Ambiental que debe realizar la Administración.

Hasta finales de 1990 se han tramitado por la Administracion Central 215 ElA, de las que un $59,1 \%$ se refieren a carreteras. Pese a haberse iniciado tal número de proyectos sólo 20 han alcanzado la Declaración de Impacto Ambiental (DIA) (un 9,3\% del total de evaluaciones iniciadas), la mayor parte de las evaluaciones se encuentran en fase de información al titular del proyecto (Figura 1).

Los proyectos tramitados por CC.AA. son mucho más numerosos (un total de 1.028), entre los que destacan los referentes a proyectos mineros $(49,3 \%)$. El porcentaje de DIA es también más elevado, ya que asciende al $24,3 \%$ de los proyectos en los que se ha iniciado evaluación de impacto ambiental (Figura 2).

Entre las evaluaciones que han fructificado en DIA existen grandes diferencias en el tiempo de realización, que se manifiestan entre los diversos tipos de proyectos (Figura 3 ), e incluso entre evaluaciones de un mismo tipo.

Las carreteras son los proyectos que necesitan un mayor tiempo para conseguir la DIA, que se establece en 16,5 meses, mientras que en la minería se reduce a nueve meses. En el resto de proyectos (grandes presas, variantes, industrias quimicas e instalaciones radioactivas) oscilan entre 10 y 11 meses (Cuadro 1).

En cualquier caso también es preciso señalar las acusadas diferencias en el tiempo de realización de un mismo tipo de proyectos. En el caso de carreteras la oscilación es muy elevada, de 14 meses, parecida a la que se produce entre las diferentes DIA referentes a instalaciones radioactivas, que se sitúan en 13 meses.

Para el resto el rango (diferencia entre el máximo y mínimo) es menor aunque también importante (Figura 4). 

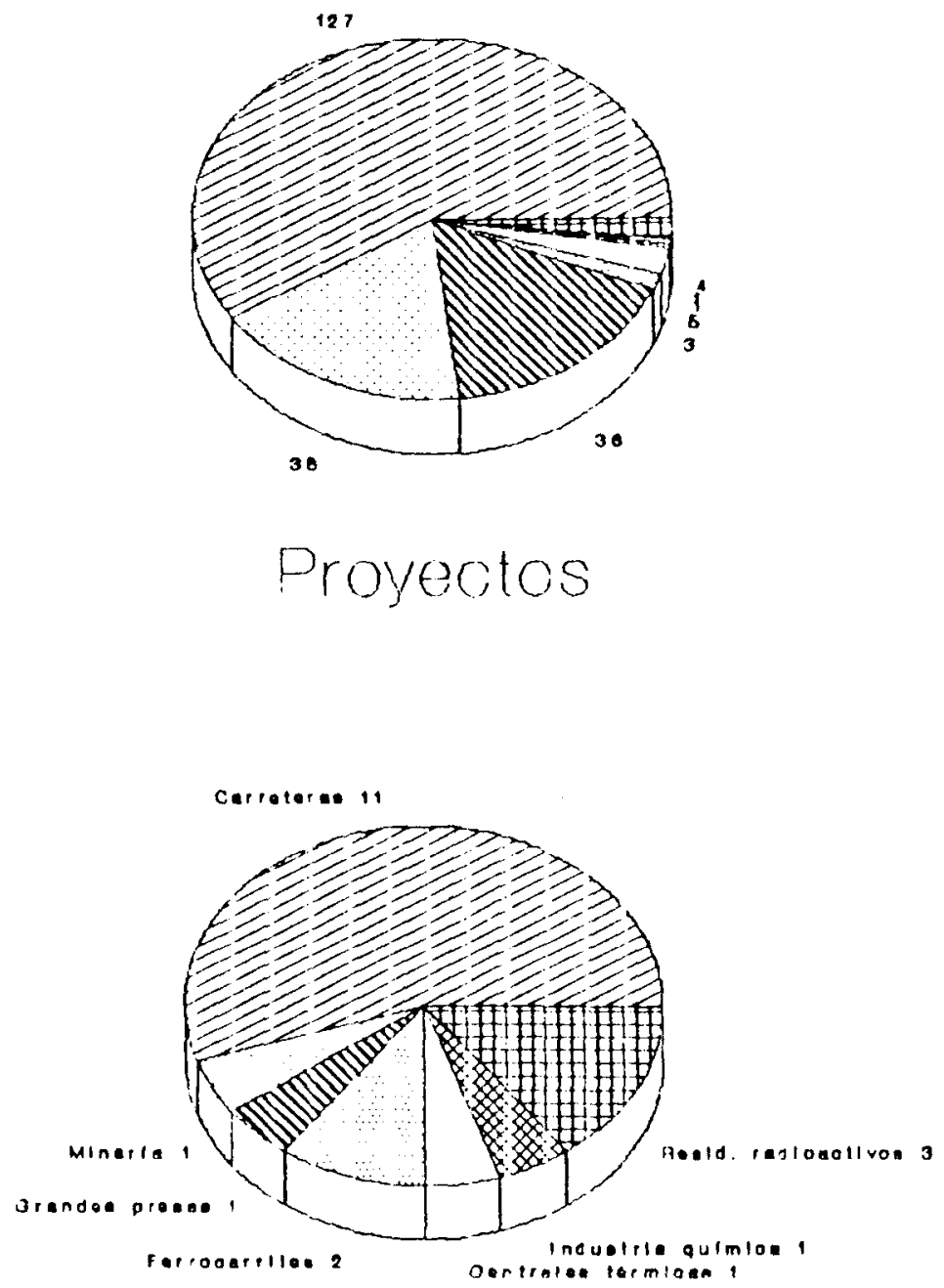

Declaración Impacto Ambiental

Fig. 1. Proyectos tramitados de ElA. Total hasta final de 1990. 


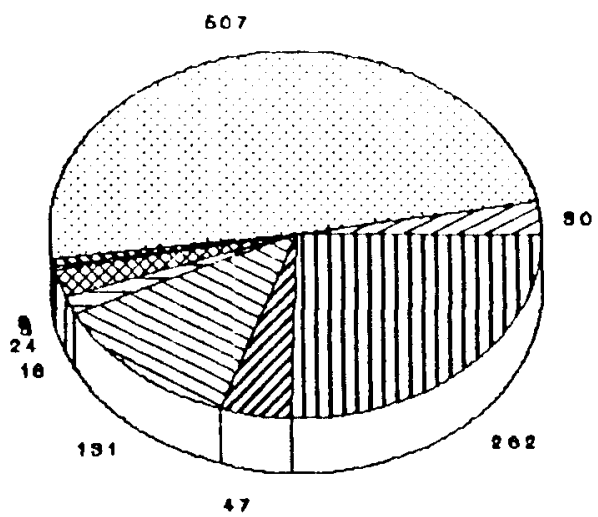

Proyectos

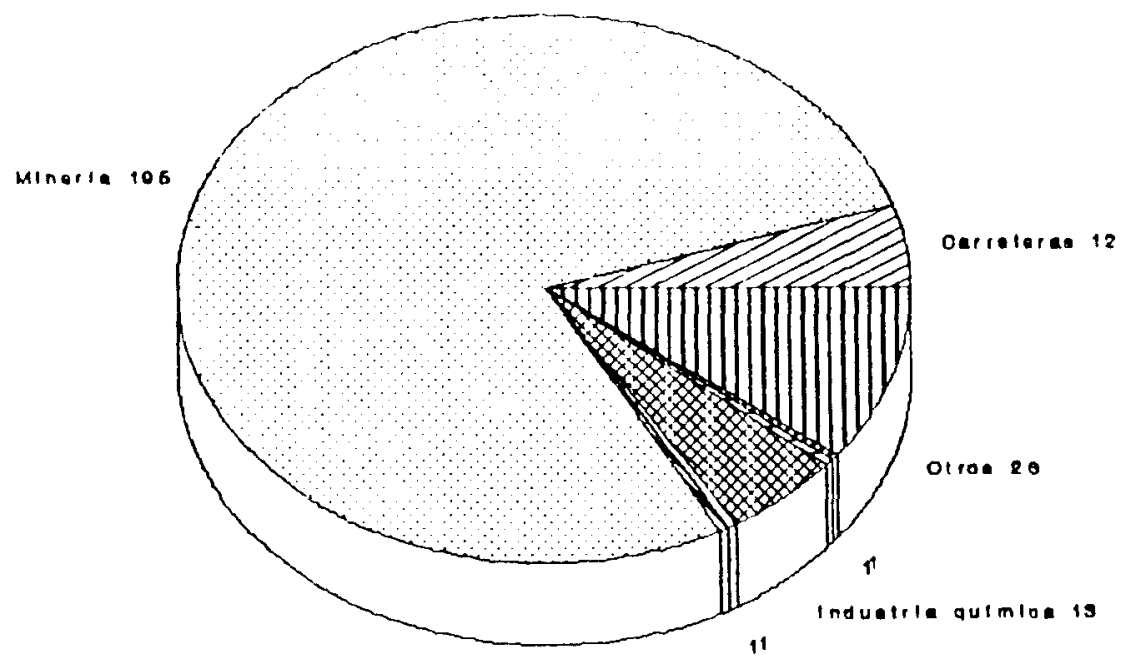

Declaración Impacto Ambiental

Fig. 2. Proyectos tramitados de EIA en las CC.AA. Total hasta final de 1990. 


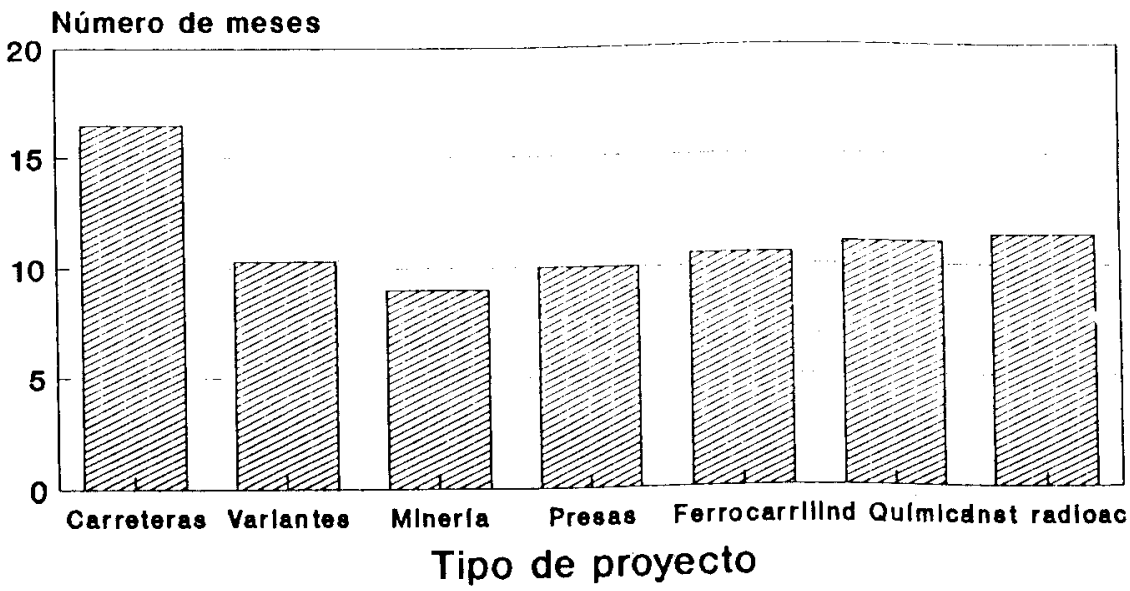

meses

Fuente: MOPT. Elaboraclón propla.

Flgura 3.

Fig. 3. Tiempo medio de los procedimientos de declaración de impacto ambiental.

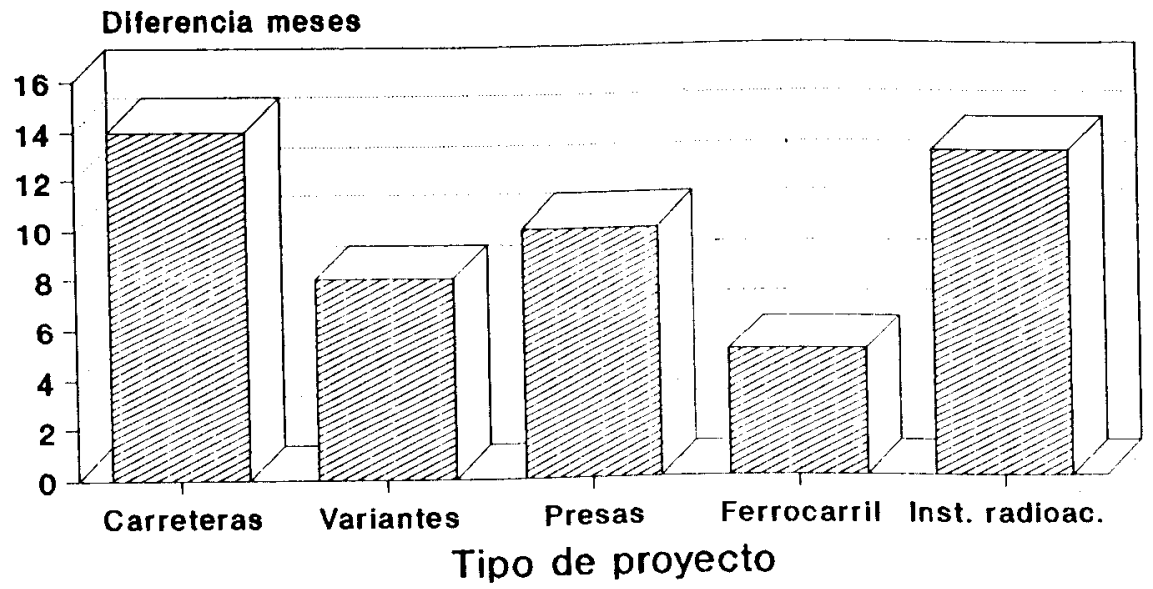

Dif. máximo-minimo

Fuente: MOPT. Elaboración propla FIgura 4.

Fig. 4. Rango de la duración de los distintos procedimientos de DIA. 
Problemas de la evaluación del impacto ambiental en relación al planteamiento...

CUADRO 1. DURACIÓN -EN MESES- DE LOS PROCEDIMIENTOS DE EVALUACIÓN AMBIENTAL CON DIA

\begin{tabular}{lccc}
\hline TIPO DE PROYECTO & DURACIÓN MEDIA & MÁXIMA & MÍNIMA \\
\hline Carreteras & 16,5 & 24 & 10 \\
Variantes & 10,3 & 13 & 5 \\
Minería & 9 & - & - \\
Grandes presas & 10 & 15 & 5 \\
Ferrocarril alta velocidad & 10,5 & 13 & 8 \\
Industria química & 11 & - & - \\
Instalaciones radioactivas & 11,3 & 19 & 6 \\
\hline
\end{tabular}

Fuente: MOPT. Elaboración propia.

Estas diferencias, aparte de la naturaleza del proyecto, obedecen a: la calidad de los equipos, la excesiva burocratización de los procedimientos y la falta de una financiación adecuada, amén de la escasa información ambiental disponible y accesible. Sería recomendable tanto para el promotor como para la propia Administración una homogeneización del tiempo de desarrollo de los diversos procedimientos, tanto en sus diversas fases, como en su conjunto.

La puesta en práctica de la EIA, por tanto, debe llevar a ciertos ajustes tanto en los procedimientos, como en la propia amplitud de los proyectos con obligación de someterse a evaluación, como se sugiere en los anexos de la directiva 85/337/CEE y como aconseja la experiencia de otros países comunitarios con mayor tradición en evaluación ambiental.

\section{CONCLUSIÓN}

Los informes de EIA son susceptibles de aplicarse a políticas, programas y planes, aunque su índice y objetivos puedan ser modificados. Incluso ciertos impactos ambientales no son evaluables a nivel de proyectos y sí acudiendo a otras categorias superiores. Diferentes tipos de impactos pueden ser evaluados en diferentes etapas. Así se puede aplicar la metodología escalonada, en cascada; desde las políticas hasta los proyectos. De esta forma se consigue una integración externa e interna de políticas, planes y proyectos mediante la EIA.

La experiencia estadounidense y de los países comunitarios con mayor tradición en estudios de evaluación ambiental indica que es posible la integración de la EIA con la política de planeamiento regional y local. Así, en Dinamarca, Alemania, Holanda e Irlanda los planes de usos de suelo o los cambios que se deseen introducir en planes regionales con el fin 
de obtener una licencia o permiso para un proyecto están sometidos a EIA.

La Directiva de EIA de la CEE recomienda aplicación a planes urbanísticos y a un número mayor de proyectos que los recogidos por el decreto de EIA español, que se puede considerar muy moderado en sus pretensiones. Aparte, la calidad de los estudios de ElA realizados en España se puede calificar de baja. Este rasgo también se presenta en el resto de países mediterráneos de la CEE, en la que no ha existido una tradición de estudios ambientales.

Por último, sería preciso considerar las externalidades positiva y negativa de un determinado proyecto, también es necesario incluir los costos de cualquier medida correctora sugerida en el estudio de EIA. Es aconsejable, asimismo, incluir umbrales a partir de los cuales sea obligatoria la realización de EIA. También es operativo que CC.AA. o ayuntamientos tengan potestad en sus respectivos ámbitos territoriales para rebajar dichos umbrales.

\section{RESUMEN}

Se analiza la necesaria relación entre Evaluación de Impacto Ambiental y planeamiento territorial y urbanístico. Para ello se evalúan la política comunitaria y la de los estados miembros de la CEE con mayor tradición en la materia, detallando como la ampliación de la Evaluación de Impacto Ambiental puede dirigirse hacia diversos sectores de la actividad económica, así como a planes de ocupación y apropiación del suelo.

Por último, se indica la trasposición de la directiva comunitaria de Evaluación de Impacto Ambiental a la legislación española y las dificultades que ha presentado su aplicación hasta el presente momento.

\section{Palabras clave}

Deterioro ambiental, evaluación de impacto ambiental, CEE-España.

\section{Summary}

In this article the relationship betwen the Environment Impact Evaluation and the land and urban planning is studied. 
Thus, the European Community and the country menbers environmental policy is analyzed.

Finally, the Environmental Impact Evaluation legislation in the EC with that spanish is compared.

\section{Key words}

Environmental degradation, Environment Impact Evaluation, EC-Spain.

\section{BIBLIOGRAFIA}

AleXANDRE, A. (1992): "Plaidoyer pour l'environment urbain”, L'Observateur de l'OCDE, n. ${ }^{\circ} 175$, auvril-mai, págs. 16-19.

ALLENDE LANDA, J. (1990): “La evaluación de impacto ambiental. Marco de referencia y aspectos relevantes a debatir", Ciudad y Territorio, n. ${ }^{\circ} 83,1 / 90$, págs. 43-66.

COMISIÓN DE LAS COMUNIDADES EUROPEAS (1990): Libro verde sobre el medio ambiente urbano. Comunicación de la Comisión al Consejo y al Parlamento. Bruselas, CCE, 59 págs. (mec).

Curso sobre evaluaciones de impacto ambiental. (1985) Madrid, MOPU, Dirección General de Medio Ambiente, 561 págs.

El Entorno urbano: políticas medioambientales para los años 90. Madrid, OCDE-MOPT, 1991, 95 páginas.

L'Etat de l'environnement dans la Communaute Europeenne, 1986. (1987). Luxembourg, Commission des Communautes Europeennes, 370 páginas.

FUENTES BODELON, F. (1985): “Marco legal y administrativo en las evaluaciones de impacto ambiental», en Curso sobre evaluaciones de impacto ambiental. Madrid, MOPU, págs. 533560 .

López Bustos, F. L. (1991): “Las ElA: Incidencia del elemento técnico y su problemática jurídica», Noticias/CEE, n. ${ }^{\circ} 82$, nov., año VII, págs. 89-103.

LóPEZ LóPEZ, A. (1990): “Programas de acción de la Comunidad Europea en materia de medio ambiente (1973-1986) antes de la adhesión de España y Portugal», en El espacio ambiental europeo. Madrid, Univ. Complutense-Instituto Nacional del Consumo, págs. 33-44.

MAB (1988): Cities and ecology. The international Experts Meeting on Ecological Approaches to Urban Planning. Moscow, UNESCO, 213 páginas.

Medio ambiente en España, 90. (1991) Madrid, MOPT, Secretaría de Estado para las Políticas del Agua y el Medio Ambiente, 338 páginas.

Pantagua Mazorra, A.; Tafancón Rico, O.P. (1990): "Comentarios sobre los polders y el nuevo urbanismo en Holanda", Ciudad y Territorio, n. ${ }^{\circ}$ 83-1, págs. 111-119.

WOOD, C.; LEE, N.; WALSH, F.; JONES, C.E. (eds.) (1990): Evaluación de impactos ambientales. Madrid, Fundación Universidad Empresa, 66 páginas.

YOLDI, L.: «Impacto ambiental y áreas verdes». Sin datar, $\sin$ fechar, 7 páginas. (mec.). 